\title{
A Modular Mobile Exergaming System With an Adaptive Behavior
}

\author{
${ }^{1}$ Ali Karime, ${ }^{1}$ Basim Hafidh, ${ }^{2}$ Wail Gueaieb, Senior Member IEEE, ${ }^{1}$ Abdulmotaleb El Saddik, IEEE Fellow \\ ${ }^{1}$ Multimedia Computing Research Laboratory (MCRLab), University of Ottawa, Ottawa, Ontario, Canada, K1N6N5 \\ ${ }^{2}$ Machine Intelligence, Robotics, and Mechatronics (MIRaM), University of Ottawa
}

\begin{abstract}
Obesity rates in the world, especially in the developed countries are alarming. This has forced scientists to consider obesity as an epidemic due to its huge negative consequences on the societies' physical and mental health. Obese Children constitute a large portion of those affected by this epidemic and researchers are striving to find solutions which can curb its spread. Exergames have emerged as promising tools that can help in the fight against obesity because it promotes physical activity through playing. In this paper, we present a mobilebased exergaming system that targets children of different ages and that aims to encourage them to do running and jumping exercises in an enjoyable manner. It currently incorporates 1 game but its modular structure enables to easily accept even more games. The system uses a novel foot interface and a heart monitor that allow interacting with a special game that can adapt to the user's performance. The preliminary evaluations with two children have shown that the system can be an effective tool that engages users into physical activity.
\end{abstract}

Keywords-exergames; obesity; tangible user interface, human-smartphone interaction

\section{INTRODUCTION}

Obesity is a social problem that is considered as a major threat to the health and well-being of current generations [1]. Obese individuals are more likely to suffer from diabetes, stroke, cardiovascular disease, and different types of cancer [2]. The lack of physical activity is a main contributing factor in the increase of obesity among the young population. Statistics suggest that obesity is dramatically increasing among children. For instance, the Canadian Institute for Health Information has revealed that obesity among children has almost tripled in the past 20 years [3], while surveys in the US have shown that $28.8 \%$ of American adolescents are overweight or at risk of being overweight [4].

Modern lifestyles are creating fewer opportunities for children and for people in general to be involved into a physical activity. For example, the prevalence of video games has tempted children to spend a large amount of their time in front of the screen. The extended playing time has caused them to lead a sedentary lifestyle, and thus created several social, mental, and physical problems for the players [5]. The proliferation of mobile devices such as smartphones and tablets has even exacerbated the problem. Nowadays, children and adults are becoming addicted to these devices [6]. Therefore, solutions must be found in order to reduce the effect of these habits on the health of the people.

Exergaming (exercise and gaming) is thought to be a good solution for reducing obesity [7]. In an exergame, the user's physical activity is the main method of interaction with the game. Researchers such as [8] and [9] have introduced numerous exergaming systems that aim to promote physical activity in a fun and joyful manner. In this paper, we present an exergaming system that uses a mobile platform to enable the user to perform jumping and running activities while interacting with a special game developed for that purpose. The system deploys a novel user interface called "SmartInsole" to detect the activity of the user and a heart monitor to capture his or her heart rates during the play. The intensity of the game is adjusted online so that it leads the user to achieve the intended heart rate that yields an effective exercise.

The remainder of the paper is organized as follows. Section 2 briefly comments on some related literature, Section 3 elaborates on the proposed method, Section 4 reveals the preliminary evaluations and Section 5 draws the conclusion and our future work.

\section{RELATED WORK}

Exergaming has become recently an attractive topic for many researchers because of the great impact it can have on the well-being of future societies [10]. For example, Sinclair [11] presented an exergame that consists of an exercise bike which is used as an input interface to an adaptive video game that can be controlled through the speed of cycling. In [12], Hafidth introduced a smart padding system called SmartPads" which enable children to construct the shape of the padding interface of their choice. Each pad within the system is integrated with various sensors that makes it able to identify the presence of a neighboring pad once connected to it. Once a particular shape is constructed, children are able to play with different computer games that promote physical activity by stepping on the proper pads.

The gaming industry has also introduced various systems that require exerting physical activity while playing with video games. One of the most popular among these tools is the DDR from Konami [13] which consists of a number of tiles that requires the users to step on them in a timely manner while listening to motivating music. Another prevalent tool is the WII Sports [14] from Nintendo which uses a tangible remote control integrated with an accelerometer that helps track the user's hand movements. With WII Sports children can play games such as tennis, boxing, baseball and, many others, by simply waving the WII controller.

With the prevalence of smartphones and tablets all over the planet, there has been an increasing focus by researchers on providing exergaming through mobile devices. Buddharaju [15] introduced an exergaming system that enables the user to 
play the popular Space Invaders game through jumping activity. The system consists of a smartphone and an Exerpad which is a regular pad that contains a number of horizontally arranged drawings for different shapes. The idea here is that the user has to move the laser cannon in the game by moving horizontally on the pad and he/she has to jump in order to shoot the aliens. Dungeon \& Swimmers [16] is an exergame that targets swimmers and aims to provide them with a fun experience during their swimming exercise. The system uses only one feedback medium, namely auditory feedback to guide the player while swimming on his/her progress in the game. The swimmer has to fight a virtual dragon based on what he or she hears through the earphones. The game consists of three different turn types: attack, defense and heal. The player can attack the dragon, defend himself or herself, or heal by swimming in a particular mode. The game progress is narrated through the auditory feedback by delivering sounds effects. The effectiveness of the system was not yet studied at that stage. Phone Row [17] encourages players to perform moderate-intensity physical activity by requiring them to do rowing movements with their arms. The software consists of a computer boat racing game that can be accessed online. The player interacts with the game using two main physical movements, namely rowing and steering movements, while holding the smartphone in his or her hands. With those movements, the user can control the movement of a virtual boat across a virtual track. $\mathrm{Lu} \mathrm{[18]} \mathrm{presented} \mathrm{the} \mathrm{first} \mathrm{phase} \mathrm{test}$ results of a multi-phase study that aims to ameliorate the fitness level in adolescents. The first phase consisted of evaluating the attitudinal changes in adolescents while being engaged in a socially interactive game. To this aim, a mobile game prototype that enables 13 exercises was proposed. The game provides social interaction by allowing the player to compete with other peers on fitness goals. All performance data is sent to a server where scores are computed. The server also allows the other peers to communicate through cheering text messages that aim to motivate the player.

In [19], we introduced the SmatInsole which is a physical activity and gait measurement system that can communicate with a computer or a mobile device via Bluetooth communication. In this paper, we use an upgraded version of our SmartInsole device to capture the performance metrics of the user while playing a mobile-based game called the "Jumpy Cow". This version of the SmartInsole has enabled the device to generate tactile feedback to the user in order to enhance the playing experience of the user and get him or her more engaged into the exercise.

\section{PROPOSED SYSTEM}

The proposed system aims to encourage children in particular and users in general to interact with mobile-based games by performing a number of exercises. The idea is to replace the traditional mobile touching interaction with other means that promote physical activity. The system consists of two SmartInsoles [19] that can be placed inside the shoes, a heart rate monitor, and a special software game that is specially developed to encourage children to perform jumping and running activities. It is worth noting that the activities herein are one dimensional in the sense that the user performs them while staying in the same position. In other words, the user will not be asked to move around the playing spot. Instead, he or she will have to move both feet off the floor either together (jumping) or in an alternating fashion (running) Figure 1 shows the underlying system architecture along with the input devices that interact with it.

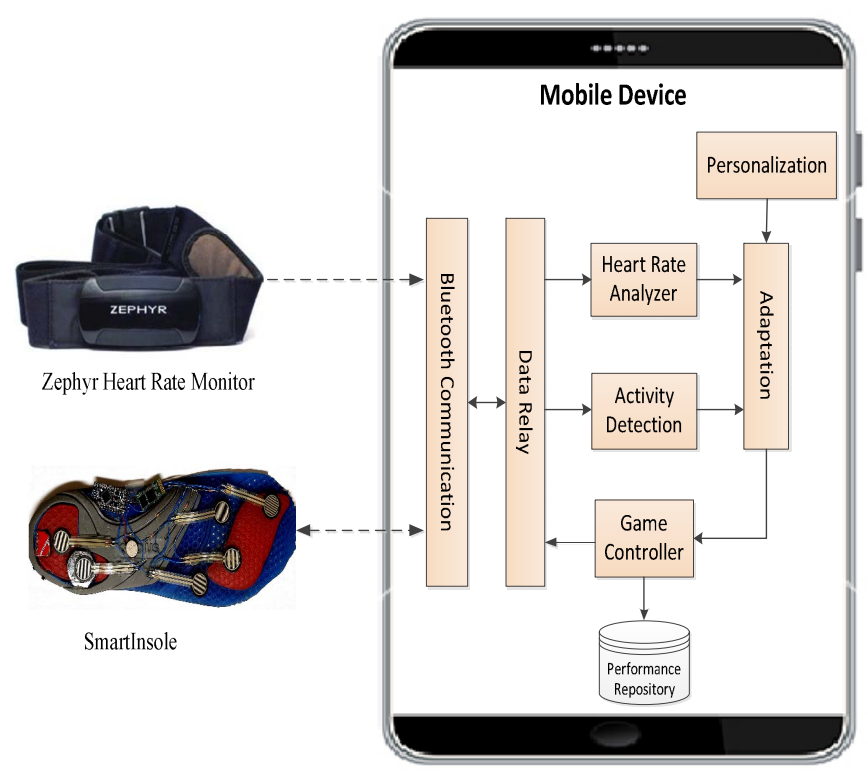

Figure 1: The proposed system architecture

\section{A. The Jumpy Cow game}

As previously mentioned, the system comprises a special game that is developed to promote physical activity in a joyful manner. Jumpy Cow is a software game designed to run on Android mobile platforms. The game was developed based on an open source game called Flappy Cow [24]. The game was implemented with Java using the Android Development Tools (ADT) which is a plugin that is installed on Eclipse IDE. The game's scenario goes as follows: a flying cow has to avoid obstacles that are encountered on the way by jumping over them. The user has to keep up with the speed of the cow during the game by moving his or her steps in a speed equal to the speed of the cow. In order to attain the required speed, the user has to move both feet on and off the floor in a faster or slower manner, therefore making more or less steps per second. In the case of higher speed requirements and if the user does not achieve the intended speed, the cow will lag behind and eventually disappear from the screen. If the speed required is less, a verbal message asking the user to slow down will be triggered. On the other hand, the user can make the cow avoid the obstacles by jumping on the floor. If the user hits a certain obstacle, the score is decreased and a tactile feedback is triggered by the SmartInsoles. A screenshot of the game is shown in Figure 2 below. 


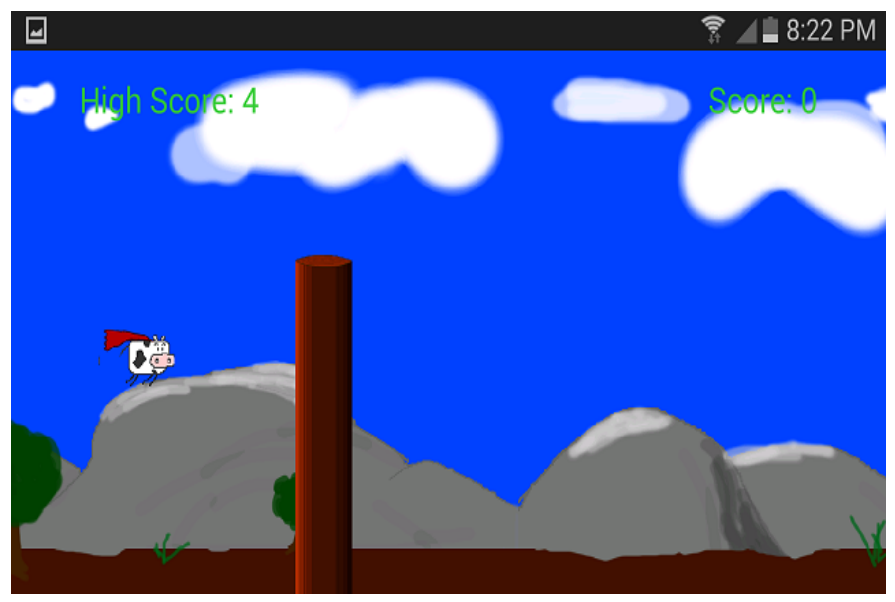

Figure 2: A screen shot of the Jumpy Cow Game

\section{B. The SmartInsole: Description}

The SmartInsole is a gait measuring tool that integrates a number of sensors that are necessary for gait and movement measurement. The insole is a highly portable system that can be used with any shoes. The system integrates the following components:

- 5 FSRs of 0.5 " sensing area diameter located on key contact points.

- An ADXL355 XYZ accelerometer located in the heel area.

- A Picaxe 14M2 microcontroller integrated with an ADC unit.

- A Linvor Bluetooth modem with 9600 baud rate.

- $3.3 \mathrm{~V}$ power supply from a rechargeable Cell battery located underneath the heel.

- Two charging inlets used to recharge the cell battery.

In order to increase the level of interaction between the user and the proposed system, haptic feedback capabilities are added to the insole. In the current version of the SmartInsole, we have integrated a $3.3 \mathrm{~V}$ vibration motor that is used to provide tactile feedback to the user. The vibration motor receives command signals during the game play at predefined events so that the user feels more immersed in the game. A detailed description of the SmartInsole can be found in [19].

\section{The Heart Rate Monitor}

The system deploys a Zephyr HxM Bluetooth Heart rate Monitor [20] that can be easily strapped with an elastic belt over the chest of the user. The device provides digital output that can be easily transmitted to the mobile device.

\section{Bluetooth Communication}

This module is responsible for providing a full-duplex communication between the hardware interface and the software game through Bluetooth communication. Its main duty is to receive and transmit the data between the smartphone and the SmartInsole, as well as the heart rate monitor.

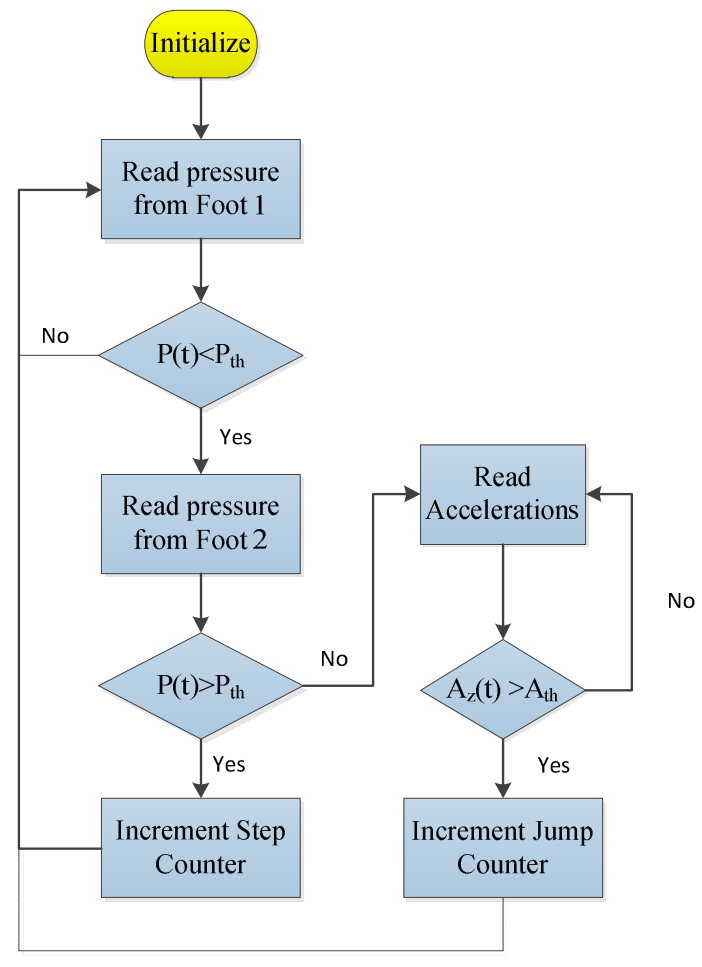

Figure 3: The activity detection algorithm

\section{E. Data Relay}

The Data Relay module tokenizes the data strings received from the input devices and relays it to the next appropriate module. It also relays the command signals received from the Game Controller to the SmartInsole.

\section{F. Activity Detection}

The Activity Detection module is responsible of analyzing the data pertaining to the physical performance of the user. It implements an algorithm that uses the acceleration and pressure information to determine if a certain activity is a "jump" or a "step". The reason why both the acceleration and the pressure are considered is because they yield better detection results when the user is performing the activity. Acceleration can indicate that the user moved his or her foot in the air while pressure would signify that the foot is actually on the floor. On the other hand, using both parameters can help the system detect if the user is trying to cheat during the game (i.e. moving feet upwards while sitting on a chair). Figure 3 shows a work flow of the algorithm implemented within this module.

Prior to the implementation of the algorithm, we have performed some tests with 15 children, age 7-12, in order to determine two particular thresholds $\mathrm{P}_{\text {th }}$ and $\mathrm{A}_{\text {th }}$. The off-floor mean pressure threshold $\left(\mathrm{P}_{\mathrm{th}}\right)$ is the mean value captured from the 6 pressure sensors when a user's foot is off the floor. On the other hand, $\left(\mathrm{A}_{\mathrm{th}}\right)$ is the acceleration value achieved during a jump. The algorithm uses the mean pressure information $(\mathrm{P}(\mathrm{t}))$ to detect a step. This is done by considering the values 
of pressure exerted by both feet at a certain time. An activity is considered a step if one foot is off the floor $\left(\mathrm{P}(\mathrm{t})<\mathrm{P}_{\mathrm{th}}\right)$ while the other is on it $\left(\mathrm{P}(\mathrm{t})>\mathrm{P}_{\mathrm{th}}\right)$. On the other hand, if the pressure detected from both feet is lower than $\mathrm{P}_{\text {th }}$ (both feet are off the floor) then we check the acceleration to verify if the user is performing a jump. If the acceleration is above $A_{t h}$ then the system assumes that the user is performing a jump with both feet in the air.

\section{G. Heart Rate Analyzer}

The Heart Rate Analyzer performs a quality check on the heart rate data captured during the play by ensuring that there are no sudden spikes or reading errors. This module may also halt the system in case of heart rates that significantly exceed the maximum rates.

\section{H. Adaptation}

This module constitutes the brain of the system since it collects the various performance data to make decisions on the intensity level of the game during the play. The objective is to encourage children to stay physically active while having fun during game play.

The adaptation engine controls mainly two parameters, the required game speed and the number of jumps to be performed per a certain time frame based on the heart rate of the user. In the jumpy cow game, this can be translated as the speed of the cow avatar which is the speed that the user should maintain and the number of obstacles per a certain time interval that the user should avoid by jumping.

Measuring the heart rate is crucial since it is a wellaccepted physical exercise intensity indicator [21]. The goal here is to constantly reduce the difference between the child's Heart Rate ( $H R$ ) and the Target Heart Rate (THR) calculated for a particular exercise intensity. This would ensure that the user is exercising at that particular intended intensity. In order to achieve that, the player's $H R$ is collected continuously through the heart rate monitor and sent wirelessly to the game. To calculate the $T H R$, the maximum Heart Rate $\left(H R_{\max }\right)$ should be calculated first. This corresponds to the highest level of a healthy heart rate during exercising [22]. $H R_{\max }$ is obtained though equation (1) [22]:

$$
H R_{\max }=A-(B \times A g e)
$$

where $A$ and $B$ are two constants equal to 206.9 and 0.67 respectively. Then using the calculated $H R_{\max }$, the $T H R$ for various exercise intensities can be obtained through equation (2) [22]:

$$
T H R=\left(H R_{\max }-H R_{\text {rest }}\right) \times I+H R_{\text {rest }}
$$

where $H R_{\text {rest }}$ is the heart rate of the player measured at rest, and $I$ is the intensity which reflects the strength of the exercise. In the system, the child can choose an intensity level between $40 \%$ to $60 \%$ of the $H R_{\max }$. The latter range covers light to moderate exercise levels [23]. Now, the velocity and obstacles frequency can be updated during the game using Equations (3) and (4).

$$
\begin{aligned}
& V_{t}=V_{t-1}+\alpha_{1}\left(T H R-H R_{t}\right) \\
& F_{t}=F_{t-1}+\alpha_{2}\left(T H R-H R_{t}\right)
\end{aligned}
$$

Here $V_{t}$ is the velocity in steps per minute, $F_{t}$ is the obstacle's frequency in obstacles per minute, and $\alpha_{1}$ and $\alpha_{2}$ are the weights of the running and jumping exercises respectively. The weights dictate how fast the velocity and the frequency are changing to keep $H R$ close to $T H R$. The values of $\alpha_{1}$ and $\alpha_{2}$ are set depending on the preference of the user. The user can choose one of three intensity levels, easy, medium and difficult, for each of the running and jumping exercises. For example, an easy level for running $\left(\alpha_{1}=0.02\right)$ and a difficult level for jumping ( $\alpha_{1}=0.1$ ) will be translated into the game by a cow that is moving slow (user has to keep up with its speed), and with the presence of many obstacles (user has to jump over).

\section{Game Controller}

The Game Engine Controller translates the data received from the Adaptation module into action by displaying the appropriate media and feedback to the user.

\section{PREliminary EVAluations}

In this section, we present our preliminary evaluations of the system which consist of evaluating both the quality of experience and the potential impact of the system on the wellbeing of the participants.

\section{A. Methods}

At this stage of the project, we invited two children, one female (participant MK) and one male (participant BS), age 8 and 11 , to play with the system at the Multimedia Communication Research Laboratory (MCRLab). The Jumpy Cow was run on a Samsung Galaxy SIII phone. Beside being paired with the smartphone, both the SmartInsoles and the Heart Rate Monitor were paired to a computer where the performance logs of the users were recorded.

\section{B. Quantitative Evaluation}

The aim of this test is to assess the potential of physical stimulation while playing with the proposed system. Since variations in heart rate (HR) correlate with exercise intensity, we adopt HR as an indicator of the level of physical activity. Figures 4 and 5 present the heart rate levels obtained over a 10 minute session. The dotted lines in the figures represent the target heart rate levels (THR), while the solid line is the heart rate of the participant over time $(\mathrm{HR}(\mathrm{t}))$. As it can be seen from the graphs, the players' HR gradually increased to stabilize in the vicinity of the target HR and almost remained at that level for the rest of the game period. 


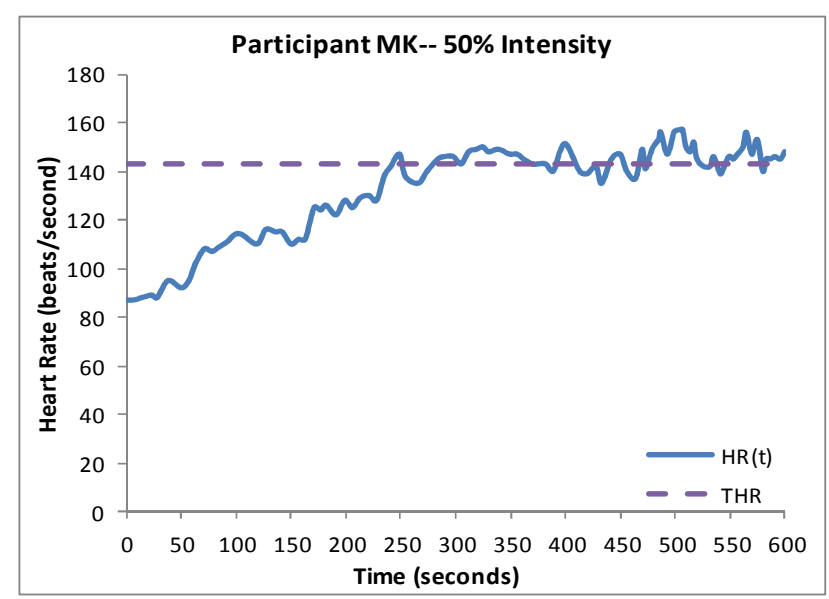

Figure 4: The heart rate pattern for participant MK

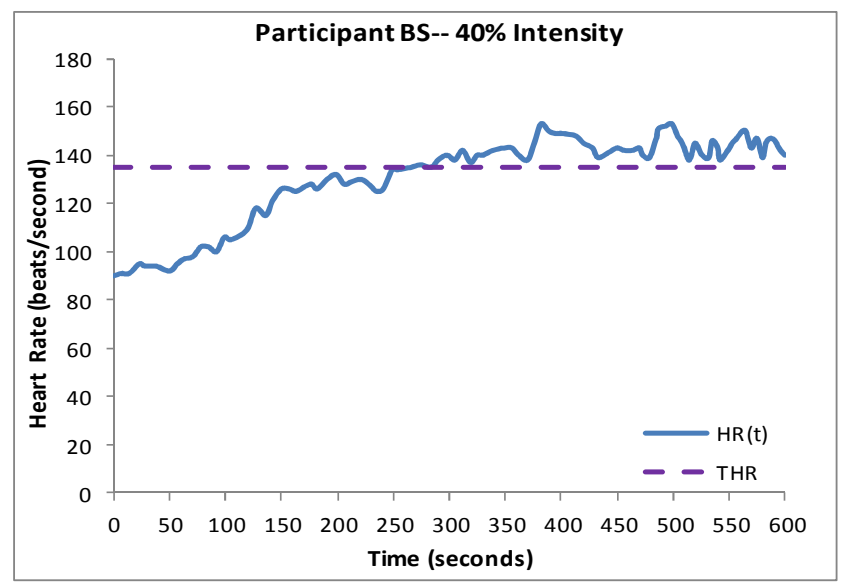

Figure 5: The heart rate pattern for participant BS

\section{Questionnaire}

In order to evaluate the quality of playing experience of the players, we asked them 5 questions at the end of the gaming sessions. The 5 questions are the following.

\section{Q1: Did you enjoy playing with Jumpy Cow?}

Q2: Do you like this way of playing the game or you prefer to play it with your fingers?

Q3: Was it hard to play with the belt on your chest?

\section{Q4: Did you feel tired while playing?}

\section{Q5: Would you suggest your friends to play with Jumpy Cow?}

Both participants have answered by "yes" for the first and second questions, while they both said that they were not bothered by the belt during the play. On the other hand, one participant said that he felt tired by the end of the playing session while the other participant said that he was a little bit tired but that he can play even more. Finally, both participants answered that they would recommend this game to their peers.

\section{CONCLUDING REMARKS AND FUTURE DIRECTIONS}

In this paper, we have presented a mobile-based exergaming system that entails the user to perform running and jumping exercises in order to interact with a software game that is specially developed to promote physical activity. The system uses the heart rate of the user to make proper adjustments to the intensity level of the game. Preliminary evaluations with two children have revealed that the system helped them achieve the required heart rate levels that would make the exercise physically effective. Our future work will include the design of a more efficient adaptation mechanism that can take into account the emotional and psychophysiological conditions of the user while making adjustment decisions. In addition, the system will be extended to include more games and to support more types of exercise, such as crouching. Finally, more thorough evaluations will be conducted with a large pool of children with different ages to examine in more details the effectiveness of the system and collect statistically more accurate data. This will help us draw more mature conclusions.

\section{REFERENCES}

[1] K. Flegal, B. Groubard, D. Williamson, and M. Gail, "Excess deaths associated with underweight, overweight, and obesity", Journal of the American Medical Association, 293(15), pp. 1861-1867, 2005.

[2] H. Strulik, "A mass phenomenon: the social evolution of obesity", Journal of Health Economics, Elsevier, 33(C), pp. 113-125, 2014.

[3] D. Spurgeon, "Childhood obesity in Canada has tripled in past 20 years", British Medical Journal, vol. 324(7351), 2002.

[4] S. M. Dorman, "Video and computer games: effect on children and implication for health education", Journal of School Health, vol.67(4), , pp.133-138, 1997.

[5] S.Yang, B.Smith, and G. Graham, "Healthy video gaming: Oxymoron or possibility?", Journal of online education, vol. 4(4), , 2008.

[6] L. Lapointe, C. Boudreau-Pinsonneault, and I. Vaghefi, "Is smartphone usage truly smart? A qualitative investigestion of IT addective behaviors", Hawaii International Conference on System Sciences (HICSS), pp. 1063-1072, 2013

[7] K. Sell, T. Lillie, and J. Taylor, "Energy expenditure during physically interactive video game playing in male college students with different playing experience", J Am Coll Health, vol. 56, no 5, pp. 505-511, 2008.

[8] S. Finkeldtein, and E. Suma, "Astrojumper: Motivating exercise with an immersive virtual reality exergame", Presence, 20(1) pp. 78-92, 2011.

[9] J. Balton, M. Lambert, D. Lirette, and B. Unsworth, "PaperDude: A vitual reality cycling exergame", CHI'14 Extended Abstracts on Human Factors in Computing Systems, pp.475-478, 2014.

[10] S. Hardy, A. El Saddik, A. Gobel, and R. Steinmetz, "Context aware serious games framework for sport and health", IEEE International Workshop on Medical Measurements and Applications (MeMeA), pp. 248-252, 2011.

[11] J. Sinclair, P. Hingston, and M. Masek, "Exergame development using the dual flow model", Proceedings on the sixth Australasian Conference on Interactive Entertainment, pp.1-7, 2009.

[12] B. Hafidth, H. Al Osman, A. Karime, J. M. Alja'am, and Abdulmotaleb El Saddik, "SmartPads: A plug-N-play configurable tangible user interface", Multimedia Tools and Applications, 72(2), pp. 1507-1530, 2014.

[13] Konami Dance Dance Revolution. http://www.konami.com/, last accessed November 2014.

[14] Nintendo, "WII Sports", http://www.nintendo.com/, last accessed November 2014. 
[15] P. Buddharaju, and N.S.C.P. Pamidi, "Mobile exergames-Burn Calories while playing games on a smartphone", IEEE Computer Vision and Pattern Recognition Workshop, pp. 50-51, 2013.

[16] H. Lee, M. Moon, T. Park, I. Hwang, U.Lee, and J. Song, "Dungeons \& swimmers: designing an interactive exergame for swimming", In Proceedings of the 2013 ACM conference on Pervasive and Ubiquitous Computing adjunct publication, pp. 287-290, 2013.

[17] M. J. Zwinderman, A. Shirzad, X.Ma, P. Bajracharya, H. Sandberg, and M. Clemens Kaptein, "Phone row: a smartphone game designed to persuade people to engage in moderate-intensity physical activity", In Persuasive Technology. Design for Health and Safety, pp. 55-66. Springer Berlin Heidelberg, 2012.

[18] F. Lu, and K.Turner, "Improving adolescent fitness attitudes with a mobile fitness game to combat obesity in youth", IEEE International Games Innovation Conference (IGIC), 2013, pp. 148-151, 2013.

[19] B. Hafidh, H. Al Osman, and A. El Saddik, "SmartInsole: A foot-based activity and gait measurement device", Multimedia and Expo Workshops, pp. 1-4, 2013.
[20] Zephyr, www.Zephyranywhere.com, last accessed Novemeber 2014.

[21] M. J. Karvonen, E. Kentala, O. Mustala, "The effects of training on heart rate: a longitudinal study", Ann Med Exper Fenn, 35(3), pp. 307$315,1957$.

[22] A. S. Jackson "Estimating Maximum Heart Rate From Age: Is It a Linear Relationship?", Medicine \& Science in Sports \& Exercise 39(5), pp. 822-829, 2007.

[23] T. C. Sell, J. P. Abt, and S. M. Lephart, "Physical activity-related benefits of walking during golf", Science and Golf V: Proceedings of the World Scientific Congress of Golf, pp. 128-132, 2008.

[24] GitHub, https://github.com/cubei/FlappyCow, last accessed November 2014. 